\title{
GLAD!
}

Revue sur le langage, le genre, les sexualités

03 | 2017

Hétérosexualités

\section{Travailler sur les hétérosexualités au prisme du langage}

Introduction

Saying and Unsaying Heterosexualities. An Introduction

Julie Abbou et Noémie Marignier

\section{OpenEdition}

\section{Journals}

Édition électronique

URL : http://journals.openedition.org/glad/861

DOI : $10.4000 /$ glad.861

ISSN : 2551-0819

Éditeur

Association GSL

Référence électronique

Julie Abbou et Noémie Marignier, "Travailler sur les hétérosexualités au prisme du langage », GLAD!

[En ligne], 03 | 2017, mis en ligne le 10 décembre 2017, consulté le 20 janvier 2021. URL : http://

journals.openedition.org/glad/861 ; DOI : https://doi.org/10.4000/glad.861

Ce document a été généré automatiquement le 20 janvier 2021.

\section{c) ()요}

La revue GLAD! est mise à disposition selon les termes de la Licence Creative Commons Attribution -

Pas d'Utilisation Commerciale - Pas de Modification 4.0 International. 


\title{
Travailler sur les hétérosexualités au prisme du langage
}

\author{
Introduction
}

Saying and Unsaying Heterosexualities. An Introduction

\author{
Julie Abbou et Noémie Marignier
}

1 Nous rédigeons cette introduction alors qu'explose la controverse sur l'écriture inclusive. Jamais les médias n'auront tant parlé de rapports entre genre et langage, ni des marques linguistiques du genre. Si dire le féminin avait déjà déclenché les foudres réactionnaires dans les années 1980 et régulièrement depuis, ce sont aujourd'hui les manières de dire le générique, la possibilité même de parler au-delà du genre ou simplement d'inclure du féminin dans le tout-masculin, qui sont sous les feux de la rampe médiatique, voire sous le feu de la vieille mitraille de l'Académie Française, qui s'étrangle ou se retourne dans sa tombe.

2 Si nous suivons de près ce chambardement discursif qui révèle un renouveau dans les évidences partagées de l'imaginaire linguistique, nous avons souhaité, avec ce premier numéro thématique de GLAD!, travailler à rebours en nous concentrant plutôt sur un impensé des rapports entre genre et langage: l'hétérosexualité. Cet impensé est double : celui de la sexualité dans les travaux en genre et langage tout d'abord, celui de l'hétérosexualité comme objet d'étude, ensuite. En effet, si le genre commence à trouver une place dans les questionnements linguistiques et plus largement langagiers, les questions de sexualités, elles, sont largement sous-représentées dans l'espace francophone. De manière plus criante encore, dans les rares travaux qui s'en préoccupent, ce n'est que très marginalement l'hétérosexualité qui est traitée.

3 L'énonciation - et ses modalités - de ce qui est mineur, silencié, ou simplement dans les marges socio-sexuelles, tout comme l'énonciation des gestes subvertissant la normativité sexuelle sont cruciales pour comprendre les mécanismes discursifs de la sexualité comme ordonnateurs des sociétés et des subjectivités, et pour réhabiliter la parole des marges. Observer ce qui se passe à la marge des normes constitue un moyen puissant de questionner les forces normatives, et par là même, de les révéler comme 
constructions sociales. Dans cette même visée, il est cependant tout aussi crucial, nous semble-t-il, de décrire empiriquement ce qui se joue au cœur des normes sexuelles, pour en déconstruire le caractère évident. C'est pourquoi nous consacrons une large part de ce numéro aux hétérosexualités, à ce qui permet de les dire comme de les dédire.

4 En effet, si les recherches sur le langage, le genre et la sexualité s'intéressent depuis longtemps aux identités lesbiennes et gaies, la question de l'hétérosexualité y a émergé, comme dans les autres disciplines (voir Tin 2008 pour les cultural studies, notamment), de manière plus discrète (on peut tout de même évoquer Cameron \& Kulick 2003; Kitzinger 2005 ; Eckert 2011). Dans l'espace francophone en particulier, rares sont les travaux qui s'interrogent sur la construction discursive des hétérosexualités (Deschamps, Gaissad \& Taraud 2009). Pourtant, l'hétérosexualité recouvre, au même titre que d'autres sexualités, un ensemble de pratiques, d'idéologies et d'identités historiquement et socialement situées: loin d'être essentielle ou naturelle, elle est configurée et reconfigurée à chaque époque au fil des interactions et de la circulationproduction des discours au sein des rapports sociaux. Impossible à penser hors du rapport qu'elle entretient avec d'autres catégories, l'hétérosexualité se présente comme un double rapport d'antagonisme : d'une part, elle n'a de sens qu'à contraster avec l'homosexualité, contraste dont Jonathan Ned Katz montre bien la dimension historique et le caractère de fausse évidence, dans son article de 1997, dont on trouvera la traduction française dans ce numéro; d'autre part, elle désigne le rapport de prétendue complémentarité entre deux entités sexuées opposées, le masculin et le féminin ou l'homme et la femme. On peut donc considérer que l'hétérosexualité a au moins autant à voir avec la sexualité qu'avec le genre, les oppositions hétéro/ homosexualité et masculin/féminin se consolidant l'une l'autre dans la constitution de la norme hétérosexuelle. Plus largement, ce sont les rapports complexes et souvent contradictoires entre les conceptualisations du genre et de la sexualité (Kosofsky 2008) que l'hétérosexualité invite à penser. Enfin, l'inexistence de l'hétérosexualité en tant qu'objet de recherche repose sur et participe à l'invisibilisation de son caractère à la fois normé et normatif, lui conférant le masque d'une normalité qu'il serait inutile de définir ou d'interroger.

5 Ce numéro de GLAD! a donc pour ambition de questionner la manière dont les hétérosexualités sont produites, reproduites mais aussi contestées dans les discours, à travers différentes constructions normatives, sémiotiques, identitaires et idéologiques, en prenant pour point de départ le caractère non essentiel de l'hétérosexualité.

\section{Normes et contre-normes de l'hétérosexualité}

6 Les recherches féministes ont mis en évidence que l'injonction à l'hétérosexualité est un puissant outil de production des rapports de genre (Rubin 1975 ; Wittig 1980), tout en laissant ouvert le débat sur l'antécédence de l'une ou des autres. Hétéronormativité et normes de genre (Coates 2013) entretiennent en effet des rapports de causalité, ou du moins de coconstruction. Dire cela, c'est dire que l'hétérosexualité produit des hétéromasculinités et des hétéroféminités. On peut dès lors étudier les manifestations discursives des différentes normes hétérosexuelles et des injonctions à l'hétérosexualité, leur incorporation ou, au contraire, leur contestation, selon 
différents contextes: les espaces médiatiques (Motschenbacher 2012), les lieux multiples de sociabilités féminines et masculines (Cameron 1997 ; Kiesling 2013), etc.

7 C'est une telle démarche qu'entreprennent deux des auteur.es du numéro, Nicolas Faynot et Vulca Fidolini. Dans deux contextes différents, respectivement Dakar et Strasbourg, ils retracent les ressources discursives et argumentatives mobilisées dans la production d'hétéromasculinités, toujours plurielles. Ces hétéromasculinités tout à la fois fondent les normes de genre sur la présupposition d'hétérosexualité, et construisent des corps genrés et des corps sexués.

8 Par ailleurs, l'hétérosexualité est aussi mise en discours dans des espaces où elle n'est plus la norme : ainsi des pratiques de passing gay, lesbien ou queer, par exemple. Quels rapports de pouvoir, et avec quels enjeux politiques et militants, sont joués ou déjoués dans l'utilisation hétérosexuelle de ces performances ou de ces ressources discursives et sémiotiques? Quelles alliances se créent ou se défont, quelles stratégies sont à l'œuvre à la frontière entre pratiques et identités? Ces questions sont au cœur de l'article de Najwa Ouguerram-Magot, qui travaille la notion de passing dans un groupe queer racisé qui mobilise tantôt la race, tantôt la sexualité dans sa construction identitaire. D'une autre manière, c'est également le propos du texte exploratoire de Luc Schicharin, qui met à l'épreuve la notion de post-straight pour discuter des possibilités d'une hétérosexualité queer.

9 La question de la pluralité des hétérosexualités comme production des identités sexuées et sexuelles (Eckert \& McConnell-Ginet 1992 ; Livia \& Hall 1997) - et donc de la pluralité des pratiques discursives et des identités hétérosexuelles - se pose ainsi audelà des logiques de normes et contre-normes. Différentes identités et pratiques hétérosexuelles concurrentes, contradictoires, alliées ou complices, coexistent en discours.

De ce point de vue, bien d'autres directions que celles rassemblées dans ce numéro restent à explorer. On pense notamment aux normes et impensés de l'hétérosexualité qui circulent dans des espaces discursifs censés a priori questionner ces injonctions. En guise de poursuite, il serait ainsi pertinent d'interroger par exemple les discours hétéronormatifs (comme les discours proféministes) qui peuvent traverser les milieux féministes et militants, de s'intéresser à leurs arguments (explicites ou implicites) et à leur réception positive ou critique. On pense également à certaines pratiques et certains discours LGBTQI qui peuvent être réinvestis dans des mouvements d'appropriation par des hétérosexuel-les, par exemple la pratique du coming out hétéro dans certains espaces, notamment féministes ou LGBTQI (Mondada \& Oloff 2015), jouant ou non du privilège des marges, ou inversement la queerisation d'espaces hétérosexuels (Visser 2008). Ou encore aux hiérarchies qui sont produites entre les différentes pratiques hétéros (Rubin 1984) et à la catégorisation des hétérosexualités considérées comme déviantes, ainsi qu'aux stratégies discursives qui permettent de déjouer ces hiérarchies ou, au contraire, de s'y conformer. De même, l'analyse des discours du désir et du plaisir (par exemple dans la pornographie) hétérosexuels, mainstream ou non, pourrait constituer une intéressante perspective de recherche. Enfin, plus largement, on pourrait questionner les manières dont l'hétérosexualité, aux niveaux épistémologique et méthodologique, travaille les études sur le langage, où elle opère aussi souvent comme un impensé ou une évidence. Il en va ainsi à la fois du postulat usuel de l'hétérosexualité des enquêtées/sujets de discours comme des enquêteurices (Committee on Lesbian and Gay Concerns 1991), ou des questions 
généralement tues du corps hétérosexuel du chercheur ou de la chercheuse et des rapports sexuels sur le terrain (Kulick \& Willson 1995). Les différentes conceptualisations de l'hétérosexualité dans les recherches féministes et queer et les débats qui traversent ces recherches peuvent constituer un objet d'étude et de réflexion en soi. Comment peut-on faire travailler, dans les études sur le langage, des concepts tels que ceux d'hétérosexualité obligatoire (Wittig 1980), d'hétéronormativité, de matrice hétérosexuelle (Butler 1990) ou encore d'ordre hétérosexuel (Clair 2012) ?

Ces ouvertures potentielles montrent que l'hétérosexualité au prisme du discours reste un champ entier à explorer. Nous espérons que ce numéro, pour sa part, contribuera à thématiser l'émergence discursive des identités hétérosexuelles et servira de point de départ pour de futures recherches.

\section{La fabrique discursive de l'hétérosexualité}

Notre dossier thématique "Dire et dédire les hétérosexualités " se compose d'une traduction, de trois articles de recherche originaux, d'un texte exploratoire et d'une création littéraire audio-dessinée. Fidèle à l'intention d'interdisciplinarité de la revue, ce numéro croise histoire, sociologie, anthropologie et approche plasticienne pour explorer différents espaces discursifs : réputation familiale à Dakar, enquête avec des Queers non blanches à Paris, récits de soi et pratiques homosociales de jeunes Marocains en Alsace, discussion théorique de la pensée straight wittigienne ou encore science-fiction érotique.

Questionner les catégories de l'hétérosexualité ne pouvait se faire sans questionner l'histoire de leur frontière avec celles de l'homosexualité. C'est la tâche à laquelle s'attelle l'historien des sexualités Jonathan Ned Katz, auteur de L'invention de l'hétérosexualité, dans un article intitulé « Homosexuel et Hétérosexuel : les termes en question ", initialement paru en 1997 dans l'ouvrage édité par Martin Duberman, Queer Representations: Reading Lives, Reading Culture (NYU Press) et dont nous offrons ici une traduction en français réalisée par Charlotte Thevenet et Julie Abbou. Dans ce texte, Katz montre comment les catégories et les définitions qui sont associées à l'hétérosexualité et à l'homosexualité sont historiquement fluctuantes, voire contradictoires, et rappelle leur caractère extrêmement récent, puisque les deux catégories n'apparaissent qu'à la fin du xixe siècle.

Dans son article "Hétérosexualités en action. La production des masculinités prédatrices et complices ", Vulca Fidolini met à l'épreuve du terrain ethnographique "l'impact normatif [de l'hétérosexualité] sur ceux et celles qui cherchent à s'y conformer ». À partir d'une enquête avec deux groupes de jeunes Marocains installés en Alsace, et d'une discussion du concept de masculinité hégémonique, développé par Connell, Vulca Fidolini met à jour les mécanismes de construction de différentes hétérosexualités masculines, complémentaires les unes des autres. S'inscrivant dans la veine des travaux récents sur les masculinités (Gourarier, Rebuccini, Gouyon), son travail montre comment masculinité et hétérosexualité s'imbriquent. L'apport principal de l'article est ainsi la démonstration empirique de la pluralité des formes du pouvoir hétéronormatif, et des façons dont les acteurs peuvent ou non mobiliser ces différentes formes pour négocier des places sociales.

Nicolas Faynot s'intéresse lui aussi à la construction des hétéromasculinités, dans le contexte dakarois pour sa part. Dans un article original qui se focalise sur la notion de 
réputation, il retrace comment une réputation de bonne hétérosexualité est garante de celle de bonne masculinité. Cependant, cette réputation est perpétuellement négociée au cours de la vie d'un homme et les acteurs masculins doivent toujours se repositionner vis-à-vis d'elle. L'article, intitulé «Institution familiale et injonction à l'hétérosexualité à Dakar : À propos de la réputation masculine d'“aimer les femmes" ", retrace ainsi les parcours de quatre jeunes hommes aux prises avec cette réputation d'aimer les femmes. Là où Fidolini cherche à montrer la pluralité des hétéromasculinités nécessaires à l'hétéronormativité, Faynot se concentre sur la conduite de cette norme : le jeu réputationnel enjoint les hommes à séduire et avoir des relations avec des femmes, mais «ni trop, ni trop longtemps». Cela dessine alors une hétéronormativité en creux de l'asexualité, de l'homosexualité, d'une hétérosexualité débridée ou encore - selon l'âge - extra-matrimoniale.

Avec "Queers non blanches en France. Des discours inaudibles, des pratiques invisibles?", Najwa Ouguerram-Magot s'inscrit dans le projet de dédire les hétérosexualités. En questionnant la pratique du coming out sous l'angle de la racialisation, elle met en évidence une double contrainte, qu'elle nomme le «conflit d'allégeance ", à laquelle font face certaines queers racisées, qui doivent alors naviguer entre identification sexuelle et raciale. Afin de pouvoir dédire tout à la fois leur hétérosexualité et leur blanchité, les enquêtées qu'elle interviewe mettent en place "un régime de visibilité dissident ", qui peut recourir tant au passing qu'à un récit de soi tacite. À la suite de Saba Mahmood, Ouguerram-Magot propose ainsi un questionnement critique de la notion de capacité d'agir comprise comme une opposition entre subversion et soumission. Cette critique lui permet de débusquer les implicites libéraux, voire universalistes, qui peuvent imprégner les milieux queers français, quand bien même le queer s'est construit sur une opposition à un tel libéralisme.

17 Utilisant toute la liberté du format de contribution exploratoire, Luc Schicharin propose un texte qui questionne la possibilité d'être straight tout en reconnaissant la pensée straight comme «une oppression scientifique et politique » qui frappe «tous ceux qui sont dans la situation de dominés ». Au-delà de la réappropriation culturelle, est-il alors possible de partager l'analyse queer et d'en être complice? Dans « Devenir Post-straight? Auto-ethnographie queer d'un universitaire hétérosexuel », Schicharin livre une réflexion intime, sans jamais être auto-centrée, qui prend la forme d'une exploration plasticienne : peut-on faire de nos vies des laboratoires politiques queer, $\mathrm{y}$ compris lorsqu'on est un homme ayant des rapports privilégiés avec une femme? La critique queer peut-elle s'exercer dans d'autres espaces de la domination (de classe, par exemple) ? Le texte laisse apparaitre les discussions qu'il a suscitées chez les premièr.es lecteur.ices, sous une forme polyphonique, elle aussi dans un format exploratoire.

Enfin, dernière pièce au dossier, Les Quotas est le résultat d'une aventure éditoriale à multiples rebonds. À partir d'une nouvelle de science-fiction érotique dégenrée de MLS qui questionnait le contrôle chirurgical et sexuel des corps, Sujarei Tali a fait une bande-dessinée, reprenant l'univers graphique de la «BD (dé)coloniale » que nous avions publiée dans le numéro 2. Cette nouvelle bande-dessinée met en scène des individus queers dans un monde où l'activité sexuelle est étroitement contrôlée par l'Inter, le pouvoir totalitaire intergalactique en place. En préparant un texte d'accompagnement, nous avons eu envie d'assortir la bande-dessinée d'une lecture audio du texte à partir duquel elle avait été faite. Mais comment mettre en scène une 
voix dégenrée? C'est ce que nous avons tenté avec la connivence de nos meilleures phonéticiennes. Enfin, en préparant un texte qui expliquerait ce chemin, nous nous sommes rendu compte que nous, éditrices comme auteures, voyions dans cet objet multiple - texte, bédé, lecture - des choses bien différentes avec nos yeux de queers ou d'hétéro, nos âges, nos sensibilités : nous y voyions tantôt des hommes, tantôt des femmes, tantôt des queers, tantôt des questions d'infibulation, tantôt des questions de normativité sexuelle, etc. Plus nous croisions nos lectures, plus cet objet nous faisait dé-dire la sexualité que nous lisions dans le texte. La nouvelle, le fichier audio et la bande-dessinée sont donc accompagnées d'un texte généalogique qui tente de retracer ces lectures.

19 Au-delà de cette diversité d'approches et d'objets, cependant, la.e lecteur.e ne manquera pas de remarquer la présence tout au long du dossier des questions de racisation. Cela est le signe d'une intégration de plus en plus forte des approches intersectionnelles dans les travaux sur le genre et les sexualités, et c'est à ce titre un signe extrêmement positif ${ }^{1}$. Simultanément, cependant, cela souligne la quasi-absence, dans les recherches francophones sur le langage, de travaux sur des hétérosexualités blanches, thématisées comme telles (voir, pour l'espace anglophone américain, et audelà des questions de langage, Deliovsky 2005, Dyer 1997 ou Ward 2015). Ce second constat nous conduit à penser qu'il est peut-être encore trop tôt pour que soit mise en question la fabrique discursive de l'évidence intersectionnelle. La double centralité normative de l'hétérosexualité blanche la rendrait-elle trop difficile à saisir? La blanchitude de l'hétérosexualité serait-elle trop proche ou trop lointaine des chercheur.es qui la prennent pour objet? L'hétérosexualité blanche ne pourrait-elle pas être située ? Quoi qu'il en soit, cette absence est une interpellation sur la nécessité à observer de manière critique ce qui constitue le majoritaire, en tant qu'espace multiple et en tant que point de vue multi-situé.

De la même façon, si le numéro fait la part belle aux masculinités et aux queers, on peut remarquer l'absence de texte sur les hétéroféminités. S'il ne s'agit pas de généraliser ce constat, cette absence semble néanmoins refléter une tendance majoritaire des recherches actuelles. Il est tentant de voir là un tropisme de la pensée critique à saisir l'accumulation de traits de domination, ou de marginalisation, qui vont dans le même sens. Cela se justifie par une nécessité socio-politique de rendre possible la parole de celleux qui connaissent une double - voire triple - peine de domination. Il est pourtant nécessaire de travailler sur les divergences que connaissent les individus dans leur rapport à la domination : femmes bourgeoises, queers blancs, hommes pauvres ou enfants trans sont autant de catégories complexes qui éclairent la tension entre subjectivité et catégorisation sociale. D'autant plus que la pensée féministe a, dans d'autres époques, posé de manière critique, et au cours de nombreux débats, la question de l'hétérosexualité des femmes (chez Wittig, de Lesseps, Rich, etc.). I Love Dick ${ }^{2}$, récemment republié en anglais et traduit en français, à travers le récit autobiographique d'une femme blanche hétérosexuelle, pose justement la question de la banalité stéréotypée du désir féminin hétéro et de son expression, mais pour mieux la refuser. Ce qui peut d'abord apparaitre comme une vulgaire bluette (comme le laisse faussement entendre la quatrième de couverture de l'édition française) travaille en fait à réélaborer les termes du désir féminin hétéro et à réinventer des modes de subjectivation complexes pour les femmes blanches hétéro. L'hétérosexualité, qu'on la comprenne comme régime normatif, comme arraisonnement des femmes, ou encore 
comme espace de négociation, peut ainsi nous éclairer sur ces dynamiques complexes du rapport à la domination.

21 Ces différents points, qu'on pourrait qualifier d'actuellement " aveugles », sont révélés par le défrichage qu'effectuent les contributions de ce dossier. Les travaux sur les hétérosexualités ouvrent une brèche dans la fabrique des catégories, en révélant la complexité, la pluralité, et l'hétérogénéité des récits sexuels, permettant ainsi de revisiter le rapport du normal au normatif, mais aussi de désessentialiser l'ordre social depuis son centre. À ce titre, ce dossier, nous l'espérons, opèrera comme un déclencheur de questions, dont d'autres continueront à se saisir.

Le numéro est complété par des contributions varia. Hors dossier, dans un texte exploratoire, Julie Abbou parcourt ainsi les apports potentiels du taoïsme de Tchouangtseu pour dire ou - en écho au thème du numéro - dédire le genre. Son texte, intitulé " /Unsaying***/ Peut-on se dédire du genre ? Quelques pistes empruntées au taoïsme ", tente de tisser des liens entre la pensée du philosophe chinois et la pensée rhétorique, comme espaces de la multiplicité et du possible pour penser les rapports entre genre et langage.

La rubrique Création du volet varia accueille, elle, une nouvelle de R.J.A, « Une genre de nouvelle discrimination chromatique", qui sonde les confins des possibilités graphiques et spatiales de l'écriture du genre. Dans une frénésie (typo)graphique, R.J.A fait éclater le marquage de genre pour en faire un jeu quasi oulipien. Par l'association du ludique et de l'écriture expérimentale, cette nouvelle rappelle la nécessité de défiger nos catégories.

Ce numéro 3 comprend également la deuxième livraison des chroniques « Les genres décrits / Les genres récrits ». Daniel Elmiger nous y offre un texte jubilatoire, qui décrasse l'écriture inclusive : une "extension du domaine des signes qui font ou défont la différence». En chroniquant le texte "Une genre de nouvelle discrimination chromatique " (que l'on trouvera dans la rubrique Création), il passe en revue les procédés utilisés par l'auteur.e de ce texte. Lucy Michel, quant à elle, s'attaque au genre tel qu'il est décrit dans les grammaires pour montrer comment le caractère fortement normatif de celles-ci leur permet une valorisation toute idéologique du masculin sous couvert de technicité de la langue.

Enfin, dans la rubrique Actualités figurent les recensions de quatre ouvrages, ainsi que des résumés de thèses et de mémoires soutenus récemment en genre et langage et un compte-rendu de conférence.

\section{BIBLIOGRAPHIE}

CAMERON, Deborah. 1997. «Performing gender identity : Young men's talk and the construction of heterosexual masculinity » in Language and masculinity, JOHNSON, Sally \& MEINHOF, Ulrike Hanna (éd.). Oxford : Blackwell, 47-64. 
CAMERON, Deborah, \& KULICK, Don. 2003. Language and sexuality. Cambridge : Cambridge University Press.

CLAIR, Isabelle. 2012. « Le pédé, la pute et l'ordre hétérosexuel » Agora débats/jeunesses (60), 6778.

COATES, Jennifer. 2013. «The discursive production of everyday heterosexualities » Discourse \& Society [En ligne], 24(5), consulté le 15 décembre 2017. https://doi.org/ $10.1177 / 0957926513486070$

COMMITTEE ON LESBIAN AND GAY CONCERNS OF AMERICAN PSYCHOLOGICAL ASSOCIATION. 1991. « Avoiding heterosexual bias in language » American Psychologist [En ligne], 46(9), consulté le 15 décembre 2017. https://doi.org/10.1037/0003-066X.46.9.973

DELIOVSKY, Katerina. 2005. « Compulsory “White” Heterosexuality : The Politics of Racial and Sexual Loyalty » Socialist Studies/Études Socialistes [En ligne], 1(2), consulté le 15 décembre 2017. http://dx.doi.org/10.18740/S48C85

DESCHAMPS, Catherine, GAISSAD, Laurent \& TARAUD, Christelle (éd.). 2009. Hétéros : discours, lieux, pratiques. Paris : EPEL.

DYER, Richard. 1997. White. London : Routledge

ECKERT, Penelope. 2011. « Language and power in the preadolescent heterosexual market » American Speech 86(1), 85-97.

ECKERT, Penelope \& MC CONNELL-GINET, Sally. 1992. « Think practically and look locally: Language and gender as community-based practice » Annual review of anthropology 21, 461-490.

INGRAHAM, Chrys. 1994. « The heterosexual imaginary : Feminist sociology and theories of gender » Sociological theory 12, 203-219.

GAGNON, John H. 1973. " Scripts and the coordination of sexual conduct » in Proceedings of the Nebraska Symposium on Motivation, COLE, James K. \& DIENSTBIER, Richard (éd.), Lincoln : University of Nabraska Press, 27-59.

KATZ, Jonathan N. 2001. L'Invention de l'hétérosexualité (trad. Michel Oliva, Éliane Sokol, \& Catherine Thévenet). Paris : EPEL.

KIESLING, Scott F. 2013. " Flirting and "normative" sexualities » Journal of Language and Sexuality [En ligne], 2(1), consulté le 15 décembre 2017. https://doi.org/10.1075/jls.2.1.04kie

KITZINGER, Celia. 2005. « Heteronormativity in action : Reproducing the heterosexual nuclear family in after-hours medical calls » Social Problems 52(4), 477-498.

KOSOFSKY SEDGWICK, Eve. 2008. L'Épistémologie du placard (trad. Maxime Cervulle). Paris : Éditions Amsterdam.

KULICK, Don \& WILLSON, Margaret. 1995. Taboo, Sex, Identity and Erotic Subjectivity in Anthropological Fieldwork. London : Routledge.

LIVIA, Anna \& HALL, Kira. 1997. Queerly phrased : Language, gender, and sexuality. Oxford : Oxford University Press.

MONDADA, Lorenzo \& OLOFF, Florence. 2015. « "Hetero oder homo ?” La performance d'un coming out hétérosexuel à la radio » Langage et société (152), 17-40.

MOTSCHENBACHER, Heiko. 2012. « "I think Houston wants a kiss right ?” : Linguistic constructions of heterosexualities at Eurovision Song Contest press conferences » Journal of 
Language and Sexuality [En ligne], 1(2), consulté le 15 décembre 2017. https://doi.org/10.1075/ jls.1.2.01mot

PAIZ, Joshua M. 2015. « Over the monochrome rainbow : Heteronormativity in ESL reading texts and textbooks » Journal of Language and Sexuality 4(1), 77-101.

ROLLIN, Zoé. 2012. «Genre et sexualité dans le rapport pédagogique : ethnographie d'un lycée “de banlieue" » Genre, sexualité \& société [En ligne], 7, consulté le 15 décembre 2017. https:// doi.org/10.4000/gss.2350

RUBIN, Gayle. 2008. " L'économie politique du sexe : transactions sur les femmes et systèmes de sexe/genre » Les cahiers du CEDREF [En ligne], 7, consulté le 30 décembre 2016. URL : http:// cedref.revues.org/171

RUBIN, Gayle. 2011 (1984). « Penser le sexe », in Surveiller et jouir. Pour une anthropologie politique du sexe (trad. Flora Bolter, Christophe Broqua), MESLI, Rostom (éd.), Paris : EPEL, 135-224.

TIN, Louis-George. 2008. L'invention de la culture hétérosexuelle. Paris : Éditions Autrement

VISSER, Gustav. 2008. "The homonormalisation of white heterosexual leisure spaces in Bloemfontein, South Africa » Geoforum [En ligne], 39(3), consulté le 15 décembre 2017. https:// doi.org/10.1016/j.geoforum.2007.11.004

WARD, Jane. 2015. Not Gay. Sex between Straight White Men. New York : NYU Press

WITTIG, Monique. 2007. La Pensée straight. Paris : Éditions Amsterdam.

\section{NOTES}

1. Voir dans ce sens l'article de Mélusine, récemment paru dans Panthère Première \#1: http:// pantherepremiere.org/le-dilemme-de-cologne.html

2. KRAUS, Chris. 2016. I Love Dick (trad. Alice Zeniter). Paris : Flammarion.

\section{INDEX}

Mots-clés : hétérosexualité, idéologie, normes

Keywords : heterosexuality, ideology, norms

\section{AUTEURS}

\section{JULIE ABBOU}

Titulaire d'un doctorat de Sciences du Langage (Aix-Marseille Université) sur les modifications du genre linguistique pour des motifs politiques, Julie Abbou mène des recherches sur les apports théoriques réciproques des études de genre et des sciences du langage. Elle travaille également sur les dimensions sémiotiques du genre grammatical, ainsi qu'en rhétorique sur le traitement du genre dans différents types de discours. Récemment, elle a co-dirigé l'ouvrage Gender, 
Language and the Periphery. Grammatical and social gender from the margins (John Benjamins) et publié des articles dans des revues telles que Semen, Mots les langages du politique, Current issues in Language Planning, etc.

\section{NOÉMIE MARIGNIER}

Noémie Marignier est docteure en Sciences du Langage et Attachée Temporaire d'Enseignement et de Recherche à l'université Paris 3 Sorbonne Nouvelle. Ses travaux de recherche portent sur l'articulation entre corps/sexe/sexualité et discours. Elle a soutenu en 2016 une thèse en analyse du discours intitulée Les matérialités discursives du sexe. La construction et la déstabilisation des évidences du genre dans les discours sur les sexes atypiques. Elle est membre associée du laboratoire Clesthia (Université Paris 3 Sorbonne Nouvelle) ainsi que de Pléiade (Université Paris 13 Sorbonne Cité). 